\title{
Binary Scientific Star Coauthors Core Size
}

\author{
Marcel AUSLOOS ${ }^{1,2, *}$ \\ ${ }^{1}$ rés. Beauvallon, rue de la Belle Jardinière, 483/0021, \\ B-4031 Liège-Nagleur, Wallonia-Brussels Federation \\ 2 "Affiliated researcher", eHumanities group \\ Royal Netherlands Academy of Arts and Sciences \\ Joan Muyskenweg 25, 1096 CJ Amsterdam, The Netherlands \\ e-mail address: marcel.ausloos@ulg.ac.be
}

June 29, 2021

\begin{abstract}
It is examined whether the relationship $J \propto A / r^{\alpha}$, and the subsequent coauthor core notion (Ausloos 2013), between the number $(J)$ of joint publications (JP) by a "main scientist" (LI) with her/his coauthors (CAs) can be extended to a team-like system. This is done by considering that each coauthor can be so strongly tied to the LI that they are forming binary scientific star (BSS) systems with respect to their other collaborators. Moreover, publications in peer review journals and in "proceedings", both often thought to be of "different quality", are separetely distinguished. The role of a time interval for measuring $J$ and $\alpha$ is also examined. New indirect measures are also introduced.

For making the point, two LI cases with numerous CAs are studied. It is found that only a few BSS need to be usefully examined. The exponent $\alpha$ turns out to be "second scientist" weakly dependent, but still "size" and "publication type" dependent, according to the number of CAs or JP. The CA core value is found to be (CA or JP) size and publication type dependent, but remains in an understandable range. Somewhat unexpectedly, no special qualitative difference on the binary scientific star $\mathrm{CA}$ core value is found between publications in peer review journals and in proceedings.

In conclusion, some remark is made on partner cooperation in BSS teams. It is suggested that such measures can serve as criteria for distinguishing the role of scientists in a team.
\end{abstract}

${ }^{*}$ previously at GRAPES@SUPRATECS, ULG, Sart-Tilman, B-4000 Liège, Euroland 


\section{Introduction}

In (Ausloos 2013), it was found out that a Zipf-like law

$$
J \propto 1 / r,
$$

exists, between the number $(J)$ of joint publications of a "main scientist", called for short "leading investigator" (LI) with her/his coauthor (CA), when both the number $J$ and of CAs are "large"; $r(=1, \ldots)$ is an integer allowing some hierarchical ranking of the CAs; $r=1$ being the most prolific CA with the LI, such that $r_{M}$ is the number of CAs of a LI.

However, it was observed that a hyperbolic (scaling) law is more appropriate

$$
J=A / r^{\alpha},
$$

with $\alpha \neq 1$; usually $\alpha \leq 1$.

This finding allows one to define the core of coauthors of a scientist through a threshold (Ausloos 2013), called the $m_{a}$-index, which takes the largest $r$ possible value $\left(m_{a} \equiv r\right)$, such that $r \leq J$. This index measures the core of the most relevant coauthors in a research team, centered on the LI,. This threshold definition is analogous to that defining the $h$-index (Hirsch 2005, Hirsch 2010). Recall that the latter is a measure of the core of papers of an author, - some appropriate, though debatable, "best output" measure (Rousseau 2006, Kelly \& Jennions 2006). In this $h$-index method, one implicitly assumes that the number of "important papers" of an author, those which are the most often quoted, allows to measure the impact of a researcher (Durieux \& Gevenois 2010). However, such a citation effect is often due to the activity of a research team, centered on the LI (McDonald 1995, Melin \& Persson 1996, Kwok 2005). In fact, there has been much work on "improving" the $h$-index (Jin 2006, Jin 2007, Jin et al. 2007, Persson et al. 2004, Bornmann \& Daniel 2009, Zhang 2009), e.g., for taking into account multi authored papers, journal impact factors, etc., thereby leading to many variants (Bornmann et al. 2008, Schreiber 2010, Schreiber et al. 2012).

In contrast, the $m_{a}$ index (Ausloos 2013) measures the role of coauthors, rather than citations, and indicates the most important coworkers of a LI. Technically, one could thus measure both the relevant size and the so called strength of a research group, centered on some leader, thereby measuring some impact of research collaboration, e.g., on scientific productivity as studied in (Lee \& Bozeman 2005). The invisible college (Kretschmer 1994, Zuccala 2006) of a LI would become visible, easily quantified, whence pointing out to some criterion for some selection in the community.

Moreover, it is of common knowledge that a LI often delegates some responsibility to senior researchers in order to pursue some activity in specific fields, often, though not always, remaining in charge of each publication (Kretschmer 1985). Furthermore, there are other reasons why a LI has CAs. It can be easily thought and even argued that a LI increases his/her ease in publishing because of the many CAs. Officially, co-authorship should imply personal responsibility 
for the content of a paper. However, some "team reputation" in having either active (senior) investigators or (and) to show a large set of (junior) CAs cannot be neglected. It should be easily admitted that the (junior or senior) CAs are not full of altruism. Publishing with a well known LI brings some attention, and can be likely useful for a career (Petersen et al. 2011, Zhou et al. 2012). Conversely, some sense of "obligation", need for justifying some collaboration, pressure from another coauthor, or explicit demand, gaining favor or reciprocity, fear of offending someone or searching for some potential grant, are common factors. In view of these, one has to shock readers by recalling Hollis observation (Hollis 2001) of a negative relationship between collaboration and output. Hollis showed that for a given individual, more coauthorship is associated with higher quality, greater length, and greater frequency of publications. However, the net relationship between coauthorship and net output attributable to the individual is negative, i.e. after discounting for the number of authors. This raises the question for the need, the role, the quality, the quantity of coauthors, and what a core of CAs truly means.

Yet, undeserved authorship, assigning authorship to persons because of their authority or prestige, or as courtesy, seems much allowed: the percentage of undeserved (false) authorship has been shown to increase (Slone 1996, VuckovicDekic 2000) along with the increasing number of coauthors.

Moreover, it is somewhat commonly accepted that proceedings papers, e.g. resulting from conference presentations, are less "valuable", more easily published, and contain more coauthors than peer review journal publications. Whence, Miskiewicz (Miskiewicz 2013) recently discussed whether publications through peer review journals in contrast to so called "proceedings" 1 have some impact on the core number and on the ranking of CAs. He found also some indication concerning time intervals in a publishing list, though such differences might be attributed to the new electronic publishing means.

On the other hand, it frequently occurs that a theoretician is publishing with experimentalists and conversely, - whatever the scientific field. In that line of reasoning, Bougrine (Bougrine 2014) in a remarkably thorough paper sorted out the subfields of several scientists and discussed the subfield effects on the $m_{a}$ cores.

Therefore, a complementary question to the above investigations is hereby examined, i.e. whether a "binary scientific star"-like system is also implied in Eq.(2), or whether some deviation occurs. Thereafter, can one debate within the new above framework on such cooperation states?

The "binary scientific star" (BSS) is defined as the couple formed by the LI and one of his CAs. Their joint publications, with of course other CAs, is here examined in the spirit of studies on the "core of co-authors" (Ausloos 2013). In line with previous publications (Ausloos 2013, Miskiewicz 2013, Bougrine 2014),

\footnotetext{
${ }^{1}$ Proceedings usually contain papers resulting from presentations at scientific meetings. By including into "proceedings" (with quotation marks) papers published in encyclopedia, in vulgarization journals, or as chapters of books not related to scientific meetings, one is allowing some reasonable statistical analysis by including otherwise outliers into a sound category of papers.
} 
two active LIs list of publications with their CAs are specifically examined. For both LIs, scientific papers in peer review journals are distinguished from, but are counted with the same weight as, publications in proceedings and similar media, like encyclopedia, summer school lecture books, other book chapters, etc., - thereafter called "proceedings".

Deviations from Eq. 2) are going to be found to occur at low rank as in (Ausloos 2013, Miskiewicz 2013, Bougrine 2014). Both a king effect (Laherrère \& Sornette 1998) and a queen effect (Ausloos 2013) may exist, as indicated by an upturn at $r \leq 1$ and a horizontal curbing for $r \leq 2,3, \ldots$, respectively, in a log-log plot of the data. This at least allows to visualize the main coauthors of a LI and allows one to restrict at once the scope of the present investigations of "BSS" systems to a few CAs only, - fortunately. Various power law exponents $\alpha$ are found for Eq. 2. The $m_{a}$ value of the core of "BSS coauthors" is also examined and deduced from the numerical analysis.

Note that to look for CAs of a LI is nothing else that to measure the degree $k_{i}$ (the number of connections of a vertex) of the LI as a node $i$ in his/her scientific collaboration network (Newman 2001a, 2001b). On the other hand, to search for the distribution of CAs for a BSS is equivalent to obtaining the number of triads containing one specific link in a collaboration network. It should also appear at once that the number of different coauthors (NDCA) is equal to the highest possible rank value, $r_{M}$. Moreover, in the case of a BSS, and only in such a case, NDCA and $r_{M}$ are identical to the number of triads. Watts and Strogatz (1998) pointed out that most networks are highly clustered, in the context of social networks. This means that there is a heightened density

of triangles of acquaintances in the network. A histogram of the degrees of vertices in a real-world network often indicates a highly skewed distribution. Whence, the skewness $(s k w)$ and the kurtosis $(k r t)$ of the distributions of CAs of such BSSs will be discussed.

The methodology is briefly explained in Sect. 2. The data analysis of the coauthorship features is contained in Sect. 3. In Sect. 4, a few indirect measures are presented and discussed. In Sect. 5, some discussion on the statistical aspects of these illustrative cases are presented in line with general considerations for a LI role. Sect. 6 serves as a conclusion with some suggestion for future work, like removing constraints in the present approach, and imagining applications.

\section{Methodology}

For the following study and discussion the considered LIs are among those investigated in several previous publications (Ausloos 2013, Miskiewicz 2013, Bougrine 2014). They are called HES (H.E. Stanley) and MA (M. Ausloos) for short.

HES publication list amounts to more than 1100 "papers". Its joint publications with (many, 600 ) CAs is taken from polymer.bu.edu/hes/vitahes messina.pdf. The list has been broken into four classes: two are distinguishing 
Table 1: NP: total number of publications; NJP: number of joint publications with CAs; NJPj in journals (j); NJPp in so called "proceedings' (p), i.e. including joint publications in book chapters and in encyclopedia chapters; updated in Dec. 2012; the number of different co-authors is given as NDCA, also for that corresponding to peer review journals, NDCAj, and proceedings NDCAp

\begin{tabular}{|c||c|c|c|c|c|c|c|}
\hline \multicolumn{1}{|c||}{} & \multicolumn{7}{c|}{ publication data summary } \\
\hline & NP & NJP & NJPj & NJPp & NDCA & NDCAj & NDCAp \\
\hline HES & 1150 & 1092 & 791 & 301 & 592 & 565 & 242 \\
\hline MA & 599 & 523 & 359 & 164 & 319 & 273 & 172 \\
\hline \hline HES\&SH & 300 & 299 & 195 & 104 & 196 & 169 & 114 \\
\hline HES\&SB & 262 & 262 & 164 & 98 & 216 & 175 & 124 \\
\hline MA\&RC & 155 & 155 & 97 & 58 & 147 & 114 & 71 \\
\hline MA\&NV & 89 & 88 & 60 & 28 & 40 & 28 & 24 \\
\hline
\end{tabular}

peer review papers in scientific journals ( $\mathrm{j}$ ) from a list including (15) book chapters, 5 encyclopedia articles and papers resulting form scientific contributions at conferences, thus, see previous footnote, so called "proceedings" (p). MA has a list of about 600 publications, with $\sim 300 \mathrm{CAs}$, mixing papers in peer review journals, proceedings, encyclopedia and book chapters.

This data, updated in Dec. 2012, is concatenated in Table 1 (top), i.e. NP is the total number of publications; NJP: the number of joint publications (JP), i.e., $J$ in Eqs.(1)-(2); broken into NJPj, i.e., those in journals (j); NJPp, those in so called "proceedings" (p), thus somewhat adapting (or "generalizing") the notion of proceedings, i.e. including joint publications in book chapters and in encyclopedia chapters. In the Table, one also finds the number of different co-authors, i.e. NDCA, whence $r_{M}$. The notations are rather obvious: the publications in peer review journals or "proceedings" are called NDCAj or NDCAp, respectively.

Sometimes, some ambiguity occurs on whether a publication pertains to a scientific report presented at a conference or is a truly more elaborated paper with original findings not yet published in a peer-review journal. Indeed, several proceedings appear in scientific journal special issues. Sometimes several contributions are not truly scientific papers reporting original work, but contributions in view of vulgarization. These often involve CAs. In view of this, I took them into account as well. However, identical scientific publications have not been counted twice: for example, HES has many papers reproduced, e.g. in a compendium or in another journal.

Moreover, HES distinguishes between ( $\mathrm{j}$ and $\mathrm{p}$ ) publications before and including, 1999 from those after, and including, 2000. It was easy to break the MA publication list into such two time intervals, identical to those of HES lists, as well, also distinguishing between $\mathrm{j}$ and $\mathrm{p}$, in these time intervals. The notations, $\mathrm{j} 1, \mathrm{j} 2$, p1 and p2 are subsequently used for distinguishing time intervals. 
Table 2: Number of joint publications (NJP) of HES and MA, further broken into journals (j) or so called "proceedings" (p), with their 5 main CAs, thus making a few BSS systems

\begin{tabular}{|c||c|c|c|c|c||c|c|c|c|c|}
\hline \multicolumn{1}{|c||}{} & \multicolumn{5}{c|}{ HES \& } & \multicolumn{5}{c|}{ MA \& } \\
& SH & SB & LA & FS & CKP & RC & NV & PV & AR & HB \\
\hline NJP & 299 & 262 & 87 & 73 & 73 & 155 & 88 & 63 & 62 & 61 \\
NJPj & 195 & 164 & 40 & 48 & 37 & 97 & 60 & 42 & 39 & 32 \\
NJPp & 104 & 98 & 47 & 25 & 36 & 58 & 28 & 21 & 23 & 29 \\
\hline
\end{tabular}

They can be combined into $\mathrm{j} 1 \mathrm{p} 1, \mathrm{j} 2 \mathrm{p} 2$, and into $\mathrm{j} 1 \mathrm{j} 2 \equiv \mathrm{j}, \mathrm{p} 1 \mathrm{p} 2 \equiv \mathrm{p}$, and $\mathrm{j} 1 \mathrm{j} 2 \mathrm{p} 1 \mathrm{p} 2$ $\equiv$ jp. Thus 18 data sets are available for study. Observe that the "first" time interval spans about 30 years, the "second" about 10 years.

Later on, CAs have been counted manually and visually in each list, using a web engine http: //rainbow.arch.scriptmania.com/tools/word_counter.html. Great care, - a tedious work, was taken about CAs misspelling, and sometimes changing of name or initials with time, - in order to fully count the contribution of each CA, and not distribute it on several persons, - who are in fact only one.

\section{Display of the Data Set Numerical Content}

Having established the rules for gathering the various data to be analyzed, and obtained the latter, it seems relevant to outline scientific questions of interest emphasizing the numerical aspects used for answers. Their discussion will arise in Sect.5.

- Crude statistical aspects.

First for completeness and for further reference, let the statistical characteristics of the distributions of joint publications with all CAs, for HES and MA, as LI, both in journals (j) and in so called proceedings (p), and their "sum" be displayed. This is reported in Table 2, together with the deduced $\alpha$ and $m_{a}$ values, and their NDCA $\left(\equiv r_{M}\right)$.

- Number of "interesting" BSS.

In order to limit the number of interesting BSSs to be investigated, it seems reasonable only to consider the CAs mainly with low rank, within the king and queen effect regimes, thus much below the $m_{a}$ measure of the LI core. Such CAs are listed in Table 3, with a few characteristics publication data, i.e. for $r=1$ to 5, for HES, Havlin (SH), Buldyrev (SB), Amaral (LA), Sciortino (FS), Peng (CKP); they are followed by Ivanov (PCI), Goldberger A. (AG), Gopikrishnan (BG), and Plerou (VP), for which data is not listed here, for space savings, but they have been studied. Similarly, for MA, Cloots (RC), Vandewalle (NV), Vanderbemden 
(PV), Rulmont (AR), and Bougrine (HB) get a $r=1$ to 5 , respectively, see Table 3.

- Outstanding BSS?

In both LI cases, all these low ranking CAs have a large number of joint publications (NJP), sometimes sharing some, of course. For both LIs, however, as it readily appears, from Table 3 , two CAs much stand up over the others, i.e. their NJP $\geq 260$ or $\geq 85$, respectively, much above those with $r \geq 3$. Thus, only such two prominent CAs are investigated in the present illustration of BSS systems, i.e. SH and SB on one hand, for HES, and $\mathrm{RC}$ and $\mathrm{NV}$, on the other hand for MA.

- Detailed statistics and parameters to be found.

Thereafter, the selection of such 4 BSS cases, i.e. s HES\&SH, HES\&SB, MA\&RC and MA\&NV has been made for illustration and discussion. For completeness, the detailed data on their NP, NJP and NDCA is given in Table 1 (bottom), for these 4 BSSs. The characteristics of the distributions of each 4 BSSs, only for their whole set (jp) of joint publications with their respective CAs (and LI), are given in Table 4 . The best power law fits, through Eq.(2), lead to $\alpha$ with some $R^{2}$; each $m_{a}$ value is reported. The distribution statistical characteristics are given.

- Differences and similarities to be examined. Time dependence effects.

Tables 5-8 are summarizing data previous to questions on similarity in behavior of the BSS., They visually point to several differences to be examined next in more detail through the different subsets, see Sect. 5 . In particular, a time dependent effect on $\alpha$ and $m_{a}$ should be examined, in both types of publications here above defined.

\section{A few Indirect Measure Definitions}

As in the $h$ - index discussions, criticizing the reduction of a scientific impact, through publications citations, to a mere scalar number, it can be argued that the $m_{a}$ index has in itself the same type of defect, - reducing a team of coauthors impact to a scalar number. Therefore, along the lines of development and introduction of other indices following the $h$-index appearance, and in order of preventing such criticisms, one can also suggest other practical measures, in the CA-core index notion, considering parts or the whole $J(r)$ histogram. This would allow some some "vectorial comparison" of scientists or teams when necessary.

Indeed, the whole cumulated NJP is

$$
\sum \equiv \sum_{r=1}^{r_{M}} J_{r}
$$


Table 3: Characteristics of the distributions of HES and MA, as LI, joint publications with all CAs, in journals (j) and in so called "proceedings" (p), i.e. including joint publications in book chapters and in encyclopedia chapters, and the whole set (jp); updated in Dec. 2012. Best power law fits, Eq.(2), $m_{a}$ value, and statistical characteristics are given, illustrating the similarity in behavior

\begin{tabular}{|c||c|c|c|c|c|c|c|c|c|c|}
\hline \multicolumn{1}{|c||}{} & \multicolumn{10}{c|}{ LI distribution characteristics of CAs } \\
\hline & & $A$ & $\alpha$ & $R^{2}$ & $m_{a}$ & $\sum$ & $\mu$ & $s k w$ & $k r t$ & NDCA \\
\hline \hline HES & j & 473.41 & 0.999 & 0.914 & 20 & 2639 & 4.671 & 11.42 & 161.15 & 565 \\
\hline HES & p & 275.42 & 1.045 & 0.875 & 15 & 1243 & 5.136 & 6.281 & 47.86 & 242 \\
\hline HES & jp & 1200.57 & 1.135 & 0.868 & 26 & 3889 & 6.569 & 11.097 & 153.42 & 592 \\
\hline \hline MA & j & 205.82 & 1.029 & 0.91 & 15 & 1055 & 3.865 & 6.508 & 55.1 & 273 \\
\hline MA & p & 62.65 & 0.859 & 0.981 & 10 & 523 & 3.041 & 6.020 & 44.40 & 172 \\
\hline MA & jp & 380.28 & 1.102 & 0.903 & 19 & 1554 & 4.872 & 7.371 & 70.40 & 319 \\
\hline
\end{tabular}

in the case that the CA with rank $r$ has published $J_{r}$ publications with the LI, or with the BSS, - depending on what case is at hand. Part of the histogram can also be examined, i.e. that cumulated NJP limited to the core, as

$$
A_{a} \equiv \sum_{r=1}^{m_{a}} J_{r}
$$

The notations are reminiscent of the $A$-index (Jin 2006), in the Hirsch scientific output measurement method.

Similarly to the $h$-index literature, one can define relative indices which measure the whole surface below the empirical data of the number of joint publications, i.e.,

$$
a_{M}=\frac{1}{m_{a}} \sum_{r=1}^{r_{M}} J_{r} \equiv \frac{\sum}{m_{a}}
$$

and till the $C A$ of rank $m_{a}$, i.e., the l.h.s. part of the histogram,

$$
a_{a}=\frac{1}{m_{a}} \sum_{r=1}^{m_{a}} J_{r} \equiv \frac{A_{a}}{m_{a}} .
$$

Of course $A_{a} / \sum$, see Tables $6-8, \equiv a_{a} / a_{M}$. Obviously, $A_{a} / \sum$ gives the relative weight of the core CAs in the cumulated NJP of a LI or a BSS. Another indirect index

$$
\frac{1}{r_{M}} \sum_{r=1}^{r_{M}} J_{r} \equiv \frac{\sum}{r_{M}}
$$

is nothing else that the mean $\mu$ of the distribution 2 , i.e. the average number

\footnotetext{
${ }^{2}$ Recall that NDCA is identical to the maximum possible rank $r_{M}$, the value of $M$ depending on the case at hand
} 
Table 4: Characteristics of the distributions of each whole set (jp) of joint publications by 4 BSSs, HES\&SH, HES\&SB, on one hand, and MA\&RC, MA\&NV, on the other hand, with all their CAs; updated in Dec. 2012. The best power law fits, Eq.(2), leads to $\alpha$ values with some $R^{2}$; the deduced $m_{a}$ value from a $J$ vs. $r$ plot is reported; statistical characteristics are given, illustrating the similarity in behavior

\begin{tabular}{|c||c|c|c|c|c|c|c|c|c|c|}
\hline \multicolumn{1}{|c||}{} & \multicolumn{10}{c|}{ LI distribution characteristics of CAs } \\
\hline & & $A$ & $\alpha$ & $R^{2}$ & $m_{a}$ & $\sum$ & $\mu$ & $s k w$ & $k r t$ & NDCA \\
\hline \hline HES\&SH & jp & 272.12 & 1.074 & 0.946 & 16 & 1098 & 5.602 & 7.309 & 68.91 & 196 \\
\hline HES\&SB & jp & 260.20 & 1.064 & 0.925 & 15 & 1088 & 5.014 & 8.388 & 92.48 & 216 \\
\hline \hline MA\&RC & jp & 99.90 & 0.985 & 0.883 & 11 & 560 & 3.810 & 4.417 & 22.09 & 147 \\
\hline MA\&NV & jp & 16.35 & 0.835 & 0.860 & 5 & 104 & 2.6 & 5.329 & 28.89 & 40 \\
\hline
\end{tabular}

of JP per CA. It ranges between 2 and 6 (see Table 5). Resulting data and statistical characteristics are summarized in Tables 3-8.

In practical terms, these indirect measures are attempts to improve the sensitivity of the $m_{a}$-index to take into account the number of relevant co-authors, whatever the number of joint publications among the most frequent coauthors, whence to introduce a contrast between the most frequent CAs and the less frequent ones. Indeed in the fits, through Eq.(2), the influence of accidental or rare CAs, i.e. with large $r$ values, can be rather huge for estimating the amplitude $A$ and the exponent $\alpha$ in Eq. (2).

\section{Discussion}

Several possible ways exist in order to display and to analyze the whole data. Some difficulty arises because, as often, questions are intertwined; so are the answers.

- One can observe whether Eq.(2) holds for the 4 BSS cases when p1, p2, $\mathrm{j} 1$ and $\mathrm{j} 2$ are taken independently of each other.

- One can examine pairs like p1p2 and j1j2, i.e. comparing the evolution of scientific contributions types, in the $\mathrm{j}$ or $\mathrm{p}$ categories.

- One can examine the pairs j1p1 and $\mathrm{j} 2 \mathrm{p} 2$, thereby examining some time evolution, whatever the publication type.

- One should surely examine the case jp which encompasses all others, on both grounds, types and time.

It is obvious that a display of each item would lead to an enormous amount of figures. Instead, the whole data is summarized in Tables $4-5$ and only a few 
cases serve as an illustration through Figs. 1-7. They have been selected for their interest in showing excellent or bad fits, through the resulting $R^{2}$ value, and to illustrate at least once each of the 4 BSSs. Many other figures are available from the author upon request.

From a more general point of view, one may begin discussing the 2 parameters appearing through the power law, Eq. 22), i.e. $A$ and $\alpha$. It is usual to consider whether the fits are valid if the correlation coefficient $R^{2}$ has a large value (e.g., $\geq 0.9$ ). It is hereby confirmed that a sufficient number of CAs or JP must be examined for a given LI, if some meaningful aspect is derived from the fits. Thus, several MA\&NV cases are not further examined, and their data is not willingly reported in Table 5 .

Therefore, in the following,

- results pertaining to the time interval effect are first commented upon in Sect. 5.1 .

- followed by the statistical characteristics of the CAs distributions in Sect. 5.2 .

- the resulting core values, and finally

- the related "indirect" measures are discussed in Sect 5.3

\subsection{Time interval effects}

As a first pertinent question, let it be observed whether some stability exists as a function of time in the behaviour of BSS teams. Recall that the "first" time interval spans about 30 years, the "second" about 10 years. A summary of the analyses and results comparison can be found in the Tables. Some thorough examination leads to qualitative and quantitative observations. Whatever the time interval, one has

- a similar hierarchy holds, on all measures, for HES\&SH with respect to HES\&SB as for MA\&RC with respect to MA\&NV

- the amplitude $A$ well reflects the importance of the relative number of joint publications in all cases, whatever any occurrence of the king and queen effects.

Differences may occur depending on the time interval :

- the exponent $\alpha$ is usually close to 1 , but is slightly larger for HES than MA; however, it markedly depends on the NJP, since in a few cases it can fall to $\sim 0.7$, see the p2 HES\&SH case (Table 5), or much below, in cases not shown, because the fits are not sufficiently $R^{2}$ meaningful; yet see the MA\&NV case in Fig. 5 as such an illustration

- the exponent $\alpha$ for either $\mathrm{p} 1$ and $\mathrm{p} 2$, and for $\mathrm{j} 1$ and $\mathrm{j} 2$, for HES\&SH and HES\&SB is always larger, though not much, than for MA\&RC 
- the exponent $\alpha$ for the time interval 1, i.e. the set $\mathrm{p} 1 \mathrm{j} 1$, is larger than the $\alpha$ value for the set $\mathrm{p} 2 \mathrm{j} 2$,

- the exponent $\alpha$ for $\mathrm{p} 1$ is smaller than for the set $\mathrm{p} 1 \mathrm{j} 1$, as well as smaller than for $\mathrm{j} 1$

- the exponent $\alpha$ for $\mathrm{p} 1$ is larger than the $\alpha$ value for the set $\mathrm{j} 2$

- the exponent $\alpha$ for $\mathrm{p} 1$, for $\mathrm{j} 1$, for $\mathrm{p} 1 \mathrm{p} 2$, an for $\mathrm{j} 1 \mathrm{j} 2$ has a systematic hierarchy, for the BSS, as if there was a ranking HES\&SH, MA\&RC, HES\&SB

Therefore, the main conclusion from observing such time intervals and publication subsets leads to confirm the importance of the relative number of joint publications in all BSS cases, within a finite time interval, for obtaining $R^{2}$ meaningful values. Also the LI scientific output differences play a relatively similar role on the parameter values. Hierarchies are confirmed in each time interval for all BSS cases. Apparently these time interval studies do not indicate a JP behavior departure for the LIs or BSSs as a function of time.

\subsection{Distribution characteristics: skewness and kurtosis}

Some statistical analysis is meaningful if the distributions are not too anomalous. Therefore it is of interest to discuss higher order moments than the second. Several meaningful observations can be related to the characteristics of the distribution of joint publications with CAs, as given in the right hand side of Tables 4-5. One should observe anomalous and regular trends. For example,

- the $\mu$ values have a short range, from $\sim 2$ to $\sim 6$, for each LI and BSS, whatever the subset

- the skw values fall into a small range, about equivalent for the HES or MA cases: roughly $] 2 ; 5[$, however, with an anomalous value $\sim 7.2$ for $\mathrm{j} 1 \mathrm{j} 2$ of HES\&SH

- whence implying a large $s k w$ value for the jp, i.e. $\sim 8$

- the $s k w$ for a BSS seems to be $\sim 80 \%$ of the corresponding LI value

- the $k r t$ increases much with the number of JP, culminating in a value $\sim 83$ for $\mathrm{j} 1 \mathrm{j} 2$ of HES\&SB

- the $k r t$ ranges also much differ for HES and MA, going from $\sim 6$ till $\sim 36$ for HES, but only from $\sim 3$ till $\sim 24$ for MA; - see Table 5

- whence implying a large $k r t$ value for the jp, i.e. 90 for HES\&SB, - see Table 4

- and $\sim 30$ for MA\&NV, - see Table 4 .

- the $k r t$ for a BSS seems to be $\sim 1 / 3$ of the corresponding LI value. 
Table 5: Time interval dependence of the CA core value and other characteristics of the number of joint publication distributions in peer review journals for the 4 BSSs so examined in the text; $a_{a}=A_{a} / m_{a} ; a_{M}=\sum / m_{a}$; recall that the time interval 1 and 2 are about 30 and 10 years respectively

\begin{tabular}{|c|c|c|c|c|c|c|c|c|c|c|c|c|}
\hline & \multicolumn{2}{|c|}{ (HES \& } & \multicolumn{2}{|c|}{ (MA \& } & \multicolumn{2}{|c|}{ (HES \& } & \multicolumn{2}{|c|}{ (MA \& } & \multicolumn{2}{|c|}{ (HES \& } & \multicolumn{2}{|c|}{ (MA \& } \\
\hline & $\mathrm{SH})$ & SB) & $\mathrm{RC})$ & $\mathrm{NV})$ & $\mathrm{SH})$ & $\mathrm{SB})$ & $\mathrm{RC})$ & $\mathrm{NV})$ & $\mathrm{SH})$ & SB) & $\mathrm{RC})$ & $\mathrm{NV})$ \\
\hline & \multicolumn{4}{|c|}{ j1 } & \multicolumn{4}{|c|}{ j2 } & \multicolumn{4}{|c|}{ j1j2 } \\
\hline$m_{a}$ & 9 & 7 & 7 & 3 & 7 & 8 & 5 & - & 11 & 10 & 9 & - \\
\hline$r_{M}$ & 98 & 74 & 60 & 23 & 95 & 127 & 64 & - & 169 & 175 & 114 & - \\
\hline$A_{a}$ & 156 & 109 & 93 & 25 & 98 & 110 & 60 & - & 236 & 102 & 151 & - \\
\hline$\sum$ & 370 & 243 & 191 & 51 & 284 & 378 & 159 & - & 654 & 621 & 350 & - \\
\hline$A_{a} / \sum$ & 0.422 & 0.449 & 0.487 & 0.457 & 0.345 & 0.291 & 0.377 & - & 0.361 & 0.164 & 0.431 & - \\
\hline$a_{a}$ & 17.33 & 15.57 & 13.29 & 8.33 & 14 & 13.75 & 12 & - & 21.45 & 10.2 & 16.78 & \\
\hline$a_{M}$ & 41.1 & 34.7 & 27.3 & 17 & 40.6 & 47.25 & 31.8 & - & 59.5 & 62.1 & 38.9 & - \\
\hline
\end{tabular}

Observe that the (total, i.e. jp) distribution kurtosis for the two main BSSs with HES relative to that of the MA-BSS is very large. This is essentially due to the relatively larger NDCA in peer review journals, for the former LI, - see last line of Table 5 .

Motivated by observations and arguments by Price (1956) and others (FernándezCano et al. 2004), it has been searched whether the evolution of $s k w\left(r_{M}\right)$ and $k r t\left(r_{M}\right)$ is close to an exponential grow (or to a power law in case of scaling) for either HES and MA, but also for HES\&SH, HES\&SB, MA\&RC, and MA\&NV. In all cases, one has $R^{2} \sim 0.8$. However in view of the limited amount of data, and performing a Jake-Berra test, neither analytic form is convincing. It is observed that the mean of the distribution has an erratic behavior as a function of NJP or NDCA (i.e., $r_{M}$ ), then leading to a very low $R^{2} \sim 0.5$. Yet, $\sum$ has a fine behavior as a function of NJP or NDCA (i.e., $r_{M}$ ), then leading to a $R^{2} \sim 0.9$. The worse and best cases are shown in Fig. 8.

\subsection{Cores and Out-of-cores CAs}

The main concern of the paper stems in the meaningful existence or not of CA core values through the direct measure $m_{a}$ and the indirect ones, introduced in Sect. 4. Let us recall that these allow to distinguish the CA core and the whole CA set contributions. Thus, a discussion of the $m_{a}$ for LIs and BSSs, as well as the $A_{a}, a_{a}, a_{M}$ values are now in order.

A few points are readily obvious from the Tables. Therefore, the few points outlined here below are those which seem to imply practical considerations or are somewhat unexpected.

Recall first that $r_{M}(\mathrm{j}) \in(170,115) \geq r_{M}(\mathrm{p}) \in(120,50)$, i.e. there are more CAs in $\mathrm{j}$ than in $\mathrm{p}$, - quite contrary to any first expectation. 
Table 6: Time interval dependence of the CA core value and other characteristics of the number of joint publication distributions in proceedings for the 4 BSSs so examined in the text; $a_{a}=A_{a} / m_{a} ; a_{M}=\sum / m_{a}$; recall that the time interval 1 and 2 are about 30 and 10 years respectively

\begin{tabular}{|c|c|c|c|c|c|c|c|c|c|c|c|c|}
\hline & \multicolumn{2}{|c|}{ (HES \& } & \multicolumn{2}{|c|}{ (MA \& } & \multicolumn{2}{|c|}{ (HES \& } & \multicolumn{2}{|c|}{ (MA \& } & \multicolumn{2}{|c|}{ (HES \& } & \multicolumn{2}{|c|}{ (MA \& } \\
\hline & $\mathrm{SH})$ & SB) & $\mathrm{RC})$ & $\mathrm{NV})$ & $\mathrm{SH})$ & $\mathrm{SB})$ & $\mathrm{RC})$ & $\mathrm{NV})$ & $\mathrm{SH})$ & SB) & $\mathrm{RC})$ & $\mathrm{NV})$ \\
\hline & \multicolumn{4}{|c|}{ p1 } & \multicolumn{4}{|c|}{ p2 } & \multicolumn{4}{|c|}{ p1p2 } \\
\hline$m_{a}$ & 9 & 6 & 6 & 3 & 4 & 9 & 3 & - & 10 & 10 & 7 & 3 \\
\hline$r_{M}$ & 74 & 66 & 37 & 16 & 60 & 76 & 40 & - & 114 & 124 & 71 & 24 \\
\hline$A_{a}$ & 165 & 114 & 69 & 18 & 35 & 105 & 27 & - & 204 & 191 & 97 & 19 \\
\hline$\sum$ & 321 & 231 & 130 & 35 & 123 & 236 & 80 & - & 444 & 467 & 210 & 44 \\
\hline$A_{a} / \sum$ & 0.514 & 0.494 & 0.531 & 0.514 & 0.285 & 0.445 & 0.340 & - & 0.460 & 0.409 & 0.462 & 0.432 \\
\hline$a_{a}$ & 18.33 & 19 & 11.5 & 6 & 8.75 & 11.67 & 9 & - & 20.4 & 19.1 & 13.86 & 6.33 \\
\hline$a_{M}$ & 35.7 & 38.5 & 21.7 & 11.7 & 30.75 & 26.2 & 26.7 & - & 44.4 & 46.7 & 30 & 14.7 \\
\hline
\end{tabular}

Table 7: CA core value and other characteristics of the number of joint publication distributions in peer review journals or proceedings, and for the whole set for the (2) LIs and (4) BSSs, so examined in the text; $a_{a}=A_{a} / m_{a} ; a_{M}=\sum / m_{a}$

\begin{tabular}{|c||c|c|c|c|c|c||c|c|c|c|}
\hline \multicolumn{1}{|c||}{} & \multicolumn{3}{c|}{ HES } & \multicolumn{3}{c|}{ MA } & \multicolumn{2}{c|}{ (HES \& } & \multicolumn{2}{c|}{ (MA \& } \\
& \multicolumn{3}{l|}{} & \multicolumn{2}{c|}{} & SH) & SB) & RC) & NV) \\
\hline & $\mathrm{j}$ & $\mathrm{p}$ & $\mathrm{jp}$ & $\mathrm{j}$ & $\mathrm{p}$ & $\mathrm{jp}$ & $\mathrm{jp}$ & $\mathrm{jp}$ & $\mathrm{jp}$ & $\mathrm{jp}$ \\
\hline$m_{a}$ & 20 & 15 & 26 & 15 & 10 & 19 & 16 & 15 & 11 & 5 \\
$r_{M}$ & 565 & 242 & 592 & 273 & 172 & 319 & 196 & 216 & 147 & 40 \\
$A_{a}$ & 895 & 549 & 1625 & 482 & 221 & 810 & 524 & 469 & 280 & 52 \\
$\sum$ & 2639 & 1243 & 3389 & 1055 & 523 & 1554 & 1098 & 1088 & 560 & 104 \\
$A_{a} / \sum$ & 0.339 & 0.442 & 0.479 & 0.457 & 0.423 & 0.521 & 0.477 & 0.431 & 0.5 & 0.5 \\
$a_{a}$ & 44.75 & 36.6 & 62.5 & 32.13 & 22.1 & 42.63 & 32.75 & 31.27 & 25.45 & 10.4 \\
$a_{M}$ & 132.0 & 82.9 & 130.3 & 70.3 & 52.3 & 81.8 & 68.625 & 72.53 & 50.91 & 20.8 \\
\hline
\end{tabular}


Table 8: (lhs) Data summary about the number of joint publication distributions of a BSS made of a (LI \& one main CA), in journals (j) or "proceedings" (p), in time interval 1 or 2 (see text); $A$ and $\alpha$ are the fit parameters of Eq. (2), and $R^{2}$ the corresponding correlation coefficient. (rhs) Distribution characteristics of such joint publications: $\sum$ is the number of joint publications, - thus having at least $3 \mathrm{CA} ; \mu, s k w$, and $k r t$ are respectively the mean, skewness and kurtosis of the specific distribution; data updated from CV of HES and MA in Dec. 2012

\begin{tabular}{|c|c|c|c|c|c|c|c|c|c|c|c|}
\hline & \multicolumn{2}{|c|}{ (HES \& } & \multicolumn{2}{|c|}{ (MA \& } & & & \multicolumn{2}{|c|}{ (HES \& } & \multicolumn{2}{|c|}{ (MA \& } \\
\hline & & $\mathrm{SH})$ & SB) & $\mathrm{RC})$ & NV) & & & $\mathrm{SH})$ & SB) & $\mathrm{RC})$ & NV) \\
\hline j1 & $A$ & 74.28 & 44.77 & 38.64 & 8.85 & j1 & $\sum$ & 370 & 243 & 191 & 51 \\
\hline j1 & $\alpha$ & 0.97 & 0.94 & 0.95 & 0.79 & j1 & $\mu$ & 3.776 & 3.284 & 3.183 & 2.217 \\
\hline j1 & $R^{2}$ & 0.932 & 0.972 & 0.849 & 0.854 & j1 & skw & 4.172 & 5.155 & 2.685 & 4.092 \\
\hline $\mathrm{j} 1$ & $m_{a}$ & 9 & 7 & 7 & 3 & $\mathrm{j} 1$ & $k r t$ & 22.52 & 31.89 & 6.89 & 15.76 \\
\hline$\overline{\mathrm{j} 2}$ & $\overline{A A}$ & 40.4 & 53.5 & 22.86 & - & j2 & $\sum$ & 284 & 378 & 159 & - \\
\hline $\mathrm{j} 2$ & $\alpha$ & 0.84 & 0.86 & 0.815 & - & $\mathrm{j} 2$ & $\mu$ & 2.990 & 2.976 & 2.484 & - \\
\hline $\mathrm{j} 2$ & $R^{2}$ & 0.98 & 0.91 & 0.95 & - & j2 & skw & 4.806 & 4.230 & 3.286 & - \\
\hline $\mathrm{j} 2$ & $m_{a}$ & 7 & 8 & 5 & - & $\mathrm{j} 2$ & $k r t$ & 29.53 & 26.49 & 11.56 & - \\
\hline$\overline{\mathrm{p} 1}$ & $\bar{A}$ & 80.59 & 44.95 & 34.46 & 7.82 & p1 & $\bar{\Sigma}$ & 321 & 231 & 130 & 35 \\
\hline p1 & $\alpha$ & 1.05 & 0.963 & 1.03 & 0.85 & p1 & $\mu$ & 4.338 & 3.50 & 3.514 & 2.188 \\
\hline $\mathrm{p} 1$ & $R^{2}$ & 0.87 & 0.97 & 0.81 & 0.895 & $\mathrm{p} 1$ & skw & 3.445 & 4.142 & 1.966 & 3.034 \\
\hline $\mathrm{p} 1$ & $m_{a}$ & 9 & 6 & 6 & 3 & $\mathrm{p} 1$ & $k r t$ & 12.74 & 19.00 & 3.07 & 8.36 \\
\hline$\overline{\mathrm{p} 2}$ & 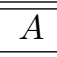 & 12.90 & 40.98 & 9.875 & - & $\overline{\mathrm{p} \text { p2 }}$ & $\bar{\Sigma}$ & 123 & 236 & 80 & - \\
\hline $\mathrm{p} 2$ & $\alpha$ & 0.676 & 0.891 & 0.676 & - & p2 & $\mu$ & 2.05 & 3.105 & 2.0 & - \\
\hline $\mathrm{p} 2$ & $R^{2}$ & 0.887 & 0.821 & 0.942 & - & p2 & skw & 5.494 & 2.423 & 3.596 & - \\
\hline $\mathrm{p} 2$ & $m_{a}$ & 4 & 9 & 3 & - & p2 & $k r t$ & 34.42 & 6.29 & 13.57 & - \\
\hline j1p1 & $A$ & 224.65 & 109.49 & 76.36 & 14.23 & j1p1 & $\sum$ & 691 & 474 & 322 & 86 \\
\hline j1p1 & $\alpha$ & 1.165 & 1.059 & 1.072 & 0.858 & j1p1 & $\mu$ & 5.906 & 4.693 & 4.076 & 2.606 \\
\hline j1p1 & $R^{2}$ & 0.865 & 0.976 & 0.786 & 0.865 & j1p1 & skw & 4.386 & 5.512 & 3.120 & 4.913 \\
\hline j1p1 & $m_{a}$ & 13 & 9 & 10 & 4 & j1p1 & $k r t$ & 23.37 & 36.05 & 9.61 & 23.87 \\
\hline $\mathrm{j} 2 \mathrm{p} 2$ & $A$ & 65.29 & 127.1 & 35.78 & - & j2p2 & $\sum$ & 407 & 614 & 239 & - \\
\hline j2p2 & $\alpha$ & 0.898 & 0.996 & 0.87 & - & $\mathrm{j} 2 \mathrm{p} 2$ & $\mu$ & 3.67 & 4.09 & 2.88 & - \\
\hline $\mathrm{j} 2 \mathrm{p} 2$ & $R^{2}$ & 0.97 & 0.856 & 0.956 & - & $\mathrm{j} 2 \mathrm{p} 2$ & skw & 5.884 & 4.296 & 4.273 & - \\
\hline j2p2 & $m_{a}$ & 9 & 11 & 6 & - & $\mathrm{j} 2 \mathrm{p} 2$ & $k r t$ & 43.81 & 26.07 & 20.70 & - \\
\hline p1p2 & $A$ & 84.6 & 88.24 & 34.92 & - & $\overline{\mathrm{p} 1 \mathrm{p} 2}$ & $\sum$ & 444 & 467 & 210 & - \\
\hline p1p2 & $\alpha$ & 0.974 & 0.969 & 0.887 & - & $\mathrm{p} 1 \mathrm{p} 2$ & $\mu$ & 3.895 & 3.766 & 2.958 & - \\
\hline p1p2 & $R^{2}$ & 0.965 & 0.944 & 0.879 & - & p1p2 & skw & 5.330 & 5.710 & 3.080 & - \\
\hline $\mathrm{p} 1 \mathrm{p} 2$ & $m_{a}$ & 10 & 10 & 7 & - & $\mathrm{p} 1 \mathrm{p} 2$ & $k r t$ & 33.95 & 42.99 & 9.30 & - \\
\hline$\overline{\mathrm{j} 1 \mathrm{j} 22}$ & $\overline{\bar{A} A}$ & $\overline{108.4}$ & 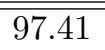 & $\overline{53.10}$ & - & $\bar{~} \overline{\mathrm{j} 1 \mathrm{j} 2}$ & $\bar{\Sigma}$ & 654 & 621 & 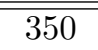 & - \\
\hline j1j2 & $\alpha$ & 0.934 & 0.922 & 0.893 & - & j1j2 & $\mu$ & 3.870 & 3.549 & 3.070 & - \\
\hline $\mathrm{j} 1 \mathrm{j} 2$ & $R^{2}$ & 0.95 & 0.92 & 0.92 & - & j1j2 & skw & 7.166 & 8.010 & 3.070 & - \\
\hline $\mathrm{j} 1 \mathrm{j} 2$ & $m_{a}$ & 11 & 10 & 9 & - & j1j2 & $k r t$ & 67.48 & 82.71 & 16.98 & - \\
\hline
\end{tabular}


Next, from Table 5, let us first distinguish a few items, i.e. for each BSS, investigating the type of publications:

- $m_{a}$ evolves expectedly when increasing the size of the investigated set, i.e. $m_{a}^{(j 1 j 2)} \geq m_{a}^{(j 1)} \geq m_{a}^{(j 2)}$,

- and $m_{a}^{(p 1 p 2)} \geq m_{a}^{(p 1)} \geq m_{a}^{(p 2)}$,

- interestingly observe a ratio reversal for $m_{a}^{(j 2 p 2)} / m_{a}^{(j 1 p 1)}$ in the case of HES\&SB, among the other BSSs, likely due to a different time for SB in joining the HES group, - as could be somewhat deduced from Tables 6-7.

Note that the $m_{a}^{(i)}$ values in the cases of BSSs are falling below the overall $m_{a}$ defining the coauthor LI-core. One can usefully compare the values in Table 3 and 4 , and observe a factor roughly $4 / 3(\sim 26 / 15 \simeq 19 / 11)$ between the two cases, somewhat measuring the relevance of the specific CA to the LI core.

Next, let us comment on indirect measures, i.e. taken from the data in Tables 6-8: a remarkable point is the similar value $a_{M}^{(j 1 j 2)}=$ e.g. from 59 to 62 ; $\simeq a_{M}^{p 1 p 2)}=$ e.g. from 44 to 47 , for the BSS with HES as LI.

Next, discuss the LI and BSS, from the (jp) point of view, see Table 8.

- $m_{a}$ has an expected hierarchy,

- likely due to the $r_{M}$ hierarchy factor

- which is reproduced in the $A_{a}$ hierarchy .

Finally "compare" LI and BSS from the core/whole set point of view, i.e. $a_{a} / a_{M}$, i.e. Table 8. First, observe that $A_{a} / \sum$ has a quasi universal value $\sim 0.5$. Unexpectedly, but remarkably, the ratio is close to $1 / 2$, - with a j-HES minor exception, where the ratio $\sim 1 / 3$.

In fine, the values can be interpreted as being due to the size of the number of CAs, i.e., $r_{M}$ or the cumulated number of joint publications, i.e. $\sum$.

It is still emphasized that very similar kings and queens occur in different publication types. The above findings suggest that $m_{a}$ is "size" dependent, fortunately.

It is also remarkable that the $a_{a}^{(j p)}$ values of the LI are almost equal to the sum of the $a_{a}^{(j p)}$ of the two BSS, so considered. This indicates that, at least in the case of HES and MA, the main two BSSs are the fully relevant items, -thus giving some weight to the approximation made in Sect. 3, according to Table 3, i.e. only considering only two BSSs for, in the present paper, such each LI.

\section{Conclusions}

The present paper aims at generalizing the coauthor core definition (Ausloos 2013) to collaborative work involving some group leader and some second in charge defined as a binary scientific star system. The paper should end with 
practical considerations based on sound findings. Let us thus successively recall ranking considerations of scientists, the present measures on teams, and conclude with suggestions.

Indeed, scientific quality is the most difficult and contentious item to measure. Often, scientific production and citations are used for quantifying scientific achievements. This has led to define a journal impact factor in illo tempore, and has recently culminated in the $h$-index for ranking individuals, journals, or teams and research centers. However, one has also questioned whether the $h$-index is the ideal way to measure research performance (Persson et al. 2004, Bornmann \& Daniel 2009).

In fact, much debate occurs on how to modify the ranking due to criteria aking into account joint publications. It is accepted that the counting, whence ranking, is sensitive to data size, while interactions and contextual variations are somewhat hidden 3 . However, it is unlikely that teamwork might be chosen for some other major reason than its effect on output.

Ausloos coauthor core definition and measure tackles such considerations in a constructive way, through the relationship between the number $(J)$ of (joint) publications with coauthors ranked according to their rank $(r)$ importance. The approach presents a great difference with respect to the Hirsch index (Hirsch 2005, 2010). The latter tests the popularity of a paper. The former emphasizes the role of persons. This seems appropriate for evaluation, and likely for weighting CAs role in quoted publications, because it is of common knowledge that a LI often delegates some responsibility to senior researchers in order to pursue some activity in specific fields, often remaining in charge of each publication, while junior workers are needed for maintaining some high publication rate. It has been here above examined what this BSS-like system implies on Eq. (1) and Eq. (2).

Thus a specific test of the findings in (Ausloos 2013), i.e., $J \propto 1 / r^{\alpha}$, has been made and discussed here above considering two prolific authors working in the field of statistical physics. It is confirmed that $\alpha \simeq 1$, also holds for BSS systems, again when $J$ and NDCA are "large", - such that the "statistics makes sense".. Interestingly, the NJP, NPmfCA, NDCA, NCA, $\alpha, R^{2}$, and $m_{a}$ values do not seem to depend on the time regime, thus on the emphasis on one or another topics, nor on the CA. Moreover, it seems that $A$ and $\alpha$ do not change much for a given author or even a given BSS as a function of time. Surprise, surprise.

The role of the main coworkers, in fact the main coauthors, has been examined, at first, supposing that publications in peer review journals and in so called proceedings might have some different influence on the core of coauthors of a LI. Indeed, on one hand, the two types of publications do not often rely on the same principles nor have the same goals or timing. No drastic difference has been seen. In fact, the number of CAs is approximately the same, be they frequent CAs or accidental CAs, in particular those occurring only once, i.e.

\footnotetext{
${ }^{3}$ Let the very interesting work on the critical mass and the dependency of research quality on group size by Kenna and Berche (2011) be mentioned here.
} 
NJP1CA. This goes against common expectation.

Observe that there is a bonus in measuring the core of CAs rather than the $h$ - index of a LI. Duplicate papers, sometimes with only cosmetic changes, are counted several times, - appearing in peer review journals and in several proceedings-like media. The true impact of the findings by such a LI should result from the (linear) addition of the quotations, but that does not seem to be done, because it requests some profound reading of the publications. In fine, it lowers the $h$-index. In contrast, the $m_{a}$ index is barely sensitive to the duplication, since the CAs are quasi the same on both types of publications on the same (or so) subject, - usually with some slight difference in the CA order.

A measure $m_{a}$ of the relevant core of coauthors thus presented in (Ausloos 2013) has thus been of interest for checking the core measure for a BSS. Practical considerations on LI and research team implications can be first observed through the NJP exponent and coauthor core value, but introduced indirect measures are also possible evaluation criteria. Some systematics has been found. Eq. (2) seems well obeyed. Note that if universal values are obviously of theoretical interest, existing deviations are of practical interest.

A final point: whether a BSS system is useful for the net output of the members of the system is an open question, following the findings of Hollis (2001). Paraphrasing Hollis, it can be stated: If society only cares about research output because of its value as a signal that the authors are competent ... , then co-authored articles may not need to be discounted. The metaphysical value of some cooperation, reciprocal altruism or manipulation, hypocrisy or prestige is reminiscent of questions on natural evolution, among which is the case of any academic career (Petersen et al. 2011, Zhou et al. 2012). How fundamental are these questions in the present framework ? ... One should conclude that the main BSSs are very relevant for a LI. It seems worthwhile to have outstanding teams, - even with LI belonging to different research centers (like HES and SH).

One may also conjecture that one can introduce selection and rewarding policies in the funding of a team (or LI) through $m_{a}$ (Ausloos 2013) and the above core and out-of-core measures. It is surely unexpected that a LI needs only a couple of CAs in order to form his/her BSS basis for publishing and attaining his/her CA core. In this respect, the effect of CA collaboration in order to increase the LI $m_{a}$ core value, as discussed in the Appendix, reveals that a cost-like matrix functional can lead to algorithmic means.

Much can still be done after the above. Three suggestions: (i) checking the deviations from a regular hyperbolic law for BSS systems, (ii) reassessing the influence of the NJP1CA, i.e. somewhat outliers, and (iii) investigating different scientific fields. Should one also consider more than two CAs, and look for ternary stars, and larger bodies within structures?

\section{Acknowledgements}

Thanks to J. Miskiewicz and H. Bougrine for private communications on (Miskiewicz 2013) and (Bougrine 2014), respectively, prior to manuscript sub-

mission. Thanks to A. Scharnhorst and S. Wyatt for comments. This paper is 
part of scientific activities in COST Action TD1210, though the paper mainly stems from the defunct COST Action MP0801.

\section{Appendix: Cost model of cumulative CA core value for a $\mathrm{LI}$}

According to Fernández-Cano et al. (2004), Statistical methods based on mere arithmetic counts are only partially adequate because any quantitative bias omits relevant qualitative features and the counting is sensitive to data size. Interactions and contextual variations are somewhat hidden. Some emphasis on group size increasing research quality by Kenna and Berche (2006) should be also mentioned here, since the study elaborates on a so called critical mass, in a similar line of thought as the present CA core measure in the main text. It was found that conclude that the best group size for experimental physicists is around 25 researchers, while in theoretical physics the number is 13. Adding more researchers to the group over these sizes does not result in an increase in research quality.

One argument about the size dependence of $m_{a}$, and other measures, may stem in the expectation that papers, sometimes with only cosmetic changes, reproduce similar results, but are counted in various ways, either cumulating references or a few being disregarded, because of the need for a limited bibliography. This is a hindrance for the $h$ - index, since similar papers are not cumulated for measuring the "quality" or "impact" of some work. Since it is accepted to prefer a quantitative approach, even if approximate, to any purely qualitative analysis, it is necessary to seek any data that can be obtained by a process of "head-counting" (Price 1956). A study of the number of coauthors and its corresponding number of joint publications goes in line with the usual knowledge that scientists who collaborate may bring additional goals to a collaboration (Sonnenwald 2003).

Thus individual goals can influence a scientist ongoing commitment to a collaboration and his or her perspective on many aspects of the work (Sonnenwald 2007). In so doing it brings much influence on the coauthorship list (Kwok 2005). However, the list of coauthors for a set of results "belonging to the same research aspects, does not change much, as seen in the main text. Thus such an influence can be thought to have some aspect of prisoner dilemma game (Bolle \& Ockenfels 1990, Bolton \& Ockenfels 2000). One may think that the resulting $m_{a}$ of all published works by a LI with his/her CAs result from a nonlinear combination of different collaborations. The result of having several CAs and/or mainly a few members of the core surely implies an increase in the $m_{a}$ value over the respective $m_{a}$ of the CAs. it is proposed that the resulting $m_{a}$ occurs through a cost function based on the "adjacency matrix" made of the individual cores. This "game model" is better understood through the following examples derived from the above results on the two examined LI and their main CAs.

From Table 4, it is known that the $m_{a}$ of HES\&SH and HES\&SB are respectively 16 and 15 . In the case of MA\&RC and MA\&NV, the $m_{a}$ values are 11 and 5 respectively. These can be taken as the values of the diagonal elements of a "cost matrix" $\mathcal{M}$ 


$$
\mathcal{M}=\left(\begin{array}{ll}
M_{11} & M_{12} \\
M_{21} & M_{22}
\end{array}\right)
$$

The off-diagonal elements are found as described in the text for the LI and the main CA independently of each other, except that the NJP intersection, between the two main coauthors of HES and MA, must be additionally searched for also. It can be found that $M_{12}=M_{21}=11$ and 3 respectively. The eigenvalues and corresponding eigenvectors of both $\mathcal{M}$ are easily obtained. They are (26.51 and 4.49) and (12.24 and 3.76) respectively.

Recall (see Table 2) that the overall HES- $m_{a}$ is equal to 26, while the MA$m_{a}$ is equal to 19. It appears that the HES- $m_{a}$ value is rather more quickly reached than the MA- $m_{a}$ value by combining, as suggested, $m_{a}$ values from the best two main CAs. This can be attributed numerically to the facts that the ratios HES- $\sum / \mathrm{MA}-\sum$, i.e. $3889 / 1554, \sim 2.50$ (see Table 2 ), and/or the ratio HES-NJP/MA-NJP, i.e. 1092/523, 2.09 (see Table 1) are large, implying a different weight of the two main CAs, thus of the two main BSSs, in the resulting

$m_{a}$ of the LI. This is an a posteriori astounding proof of the BSS role and of its effects.

\section{References}

Ausloos, M. (2013). A scientometrics law about co-authors and their ranking. The co-author, Scientometrics, 95(3), 895-909.

Bolle, F. \& Ockenfels, P. (1990). Prisoners dilemma as a game with incomplete information, Journal of Economic Psychology, 11(1), 69-84.

Bolton, G. E. \& Ockenfels, A. (2000). ERC: Equity, reciprocity and competition, American Economic Review, 90, 166-193.

Bornmann, L. \& Daniel, H.-D. (2009). The state of h index research. Is the $\mathrm{h}$ index the ideal way to measure research performance?, EMBO Reports, 10(1): 2-6.

Bornmann, L., Mutz, R. \& Daniel, H. (2008). Are there better indices for evaluation purposes than the h-index? A comparison of nine different variants of the h-index using data from biomedicine, Journal of the American Society for Information Science and Technology, 59,(5) 830-837.

Bougrine, H. (2014). Subfield Effects on the Core of Coauthors, accepted for publication in Scientometrics.

Durieux, V. \& Gevenois, P. A. (2010). Bibliometric Indicators: Quality Measurements of Scientific Publication, Radiology, 255(2), 342-351. 
Fernández-Cano, A., Torralbo, M. \& Vallejo, M. (2004). Reconsidering Price's model of scientific growth: An overview, Scientometrics, 61(3), 301-321.

Hirsch, J. E. (2005). An index to quantify an individual's scientific research output, Proceedings of the National Academy of Sciences USA, 102(46), 16569-16572.

Hirsch, J. E. (2010). An index to quantify an individual's scientific research output that takes into account the effect of multiple coauthorship, Scientometrics 85(3), 741-754.

Hollis, A. (2001). Co-authorship and the output of academic economists, Labour Economics, 8(4), 505-530.

Jin, B. (2006). h-Index: An evaluation indicator proposed by scientist, Science Focus, 1(1), 8-9.

Jin, B. (2007). The AR-index: complementing the h-index, ISSI Newsletter, $3(1), 6$.

Jin, B., Liang, L., Rousseau, R., \& Egghe, L. (2007). The R- and AR-indices: Complementing the h-index, Chinese Science Bulletin, 52(6), 855-863.

Kelly, C. D., \& Jennions, M. D. (2006). The $\mathrm{h}$ index and career assessment by numbers, Trends in Ecology and Evolution, 21(4), 167-170.

Kenna, R. \& Berche, B. (2011). Critical mass and the dependency of research quality on group size, Scientometrics, 86(2), 527-540.

Kretschmer. H. (1994). Coauthorship networks of invisible colleges and institutional communities, Scientometrics, 30(1), 363-369.

Kretschmer, H., (1985). Cooperation structure, group size and productivity in research groups, Scientometrics, 7(1), 39-53.

Kwok, L. S. (2005). The White Bull effect: abusive coauthorship and publication parasitism, Journal of Medical Ethics, 31(9), 554-556.

Laherrère J., \& Sornette, D. (1998). Stretched exponential distributions in nature and economy: fat tails with characteristic scales, European Physics Journal B, 2(4), 525-539.

Lee, S. \& Bozeman, B. (2005). The impact of research collaboration 
on scientific productivity, Social Studies of Science, 35(5), 673-702.

McDonald, Kim A. (1995). Too Many Co-Authors?, Chronicle of Higher Education, 41(33), 35-36.

Melin, G. \& Persson, O. (1996). Studying research collaboration using co-authorships, Scientometrics, 36 (3), 363-377.

Miskiewicz, J. (2013). Effects of Publications in Proceedings on the Measure of the Core Size of Coauthors, Physica A, 392(20) 5119-5131.

Newman, M. E. J., (2001a). The structure of scientific collaboration networks, Proceedings of the National Academy of Sciences USA, 98(2), 404-409.

Newman, M. E. J., (2001b). Scientific collaboration networks. I. Network construction and fundamental results, Physical Review E, 64(1), 016131 [8 pages].

Persson, O., Glänzel, W., \& Danell, R. (2004). Inflationary Bibliometric Values: The Role of. Scientific Collaboration and the Need for Relative Indicators in Evaluative Studies. Scientometrics, 60(3), 421-432.

Petersen, A. M., Jung, W.-S. , Yang, J.-S., \& Stanley, H. E. (2011) Quantitative and empirical demonstration of the Matthew effect in a study of career longevity, Proceedings of the National Academy of Sciences USA, 108(1), 18-23.

Price, D. J. de S. (1956). The exponential curve of science, Discovery, 17(6), 240-243.

Rousseau, R. (2006). New developments related to the Hirsch index, Science Focus, 1 (1): 23-25.

Schreiber, M. (2010). Twenty Hirsch index variants and other indicators giving more or less preference to highly cited papers, Annalen der Physik (Berlin), 522(8), 536-554.

Schreiber, M., Malesios, C. C. \& Psarakis, S. (2012). Exploratory factor analysis for the Hirsch index, $17 \mathrm{~h}$-type variants, and some traditional bibliometric indicators, Journal of Informetrics, 6(3), 347-358.

Slone, R. M. (1996). Coauthors contributions to major papers published in the AJR: Frequency of undeserved coauthorship, American Journal of Radiology, 167(3), 571-579. 
Sonnenwald, D.H. (2003). Expectations for a scientific collaboratory: A case study. Proceedings of the ACM GROUP 2003 Conference, pp. 68-74. NY: ACM Press.

Sonnenwald, D. H. (2007). Scientific collaboration, Annual Review of Information Science and Technology, 41(1), 643-681.

Vuckovic-Dekic., Lj. (2000). Multiauthorship - what is it, and does it matter?, Archive of Oncology, 8, 139.

Watts, D.J. \& Strogatz, S. H. (1998). Collective dynamics of smallworld networks, Nature, 393(6684), 440-442 .

Zhang, R. (2009). An index to link scientific productivity with visibility, arxiv.org/pdf/0912.3573.

Zhou, Yan-Bo, Lu, Linyuan \& Li, Menghui (2012). Quantifying the influence of scientists and their publications: distinguishing between prestige and popularity, New Journal of Physics, 14(3), 033033.

Zuccala, A. (2006). Modeling the invisible college, Journal of the American Society for Information Science and Technology, 57(2), 152-168 . 
Figure 1: Low ranking regime of the Number of Joint Publications (NJP) of two BSS made of a PI (HES or MA) with one of their two main CAs (SH or $\mathrm{SB}$, on one hand, $\mathrm{RC}$ or $\mathrm{NV}$, on the other hand) as a function of the rank of the other CAs; best fits by a power law are shown together with the line indicating the threshold on how to measure the $m_{a}$ CA core value

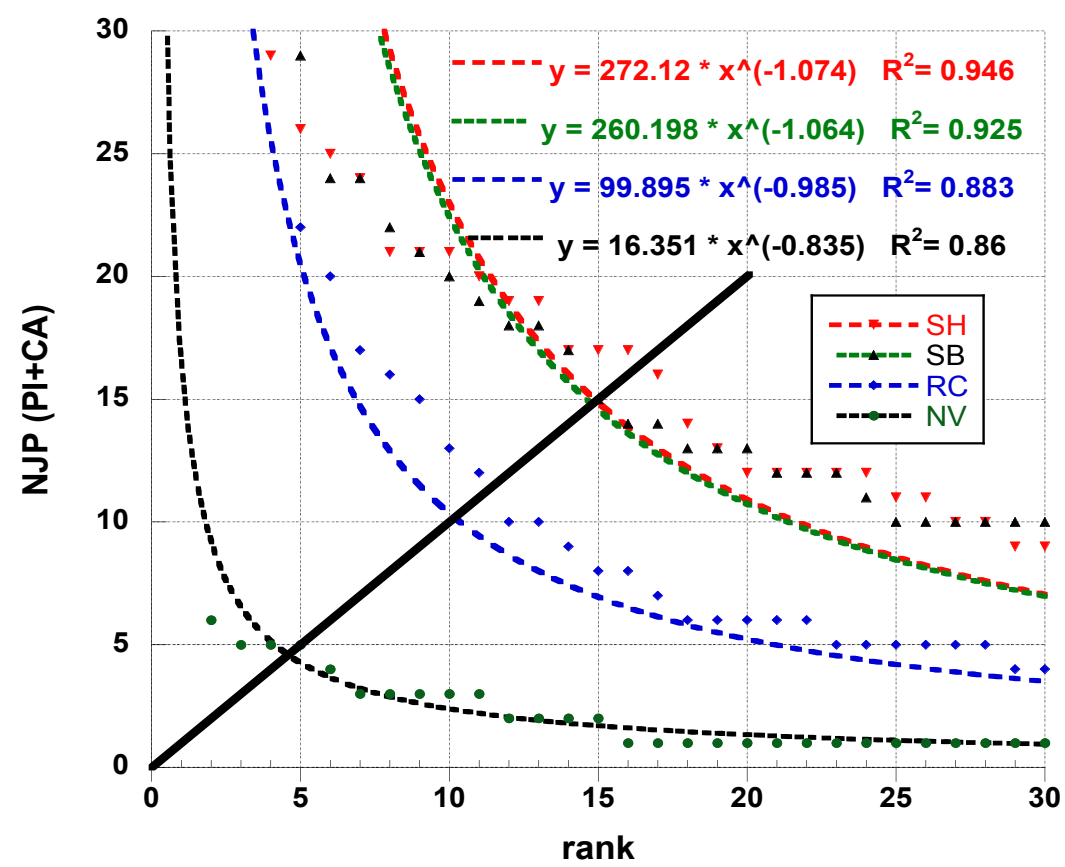


Figure 2: Number of Joint Publications (NJP) by HES\&SB as a function of the rank of CAs by decreasing importance. Best fits by a power law are shown together with the line indicating the threshold on how to measure the $m_{a} \mathrm{CA}$ core value. This allows to compare the value of Eq. 2. in time interval 1 and 2 through p1j1 and p2j2, as well as to compare the whole set of publications in proceedings (p1p2) with the whole set of papers in peer review journals $(\mathrm{j} 1 \mathrm{j} 2)$

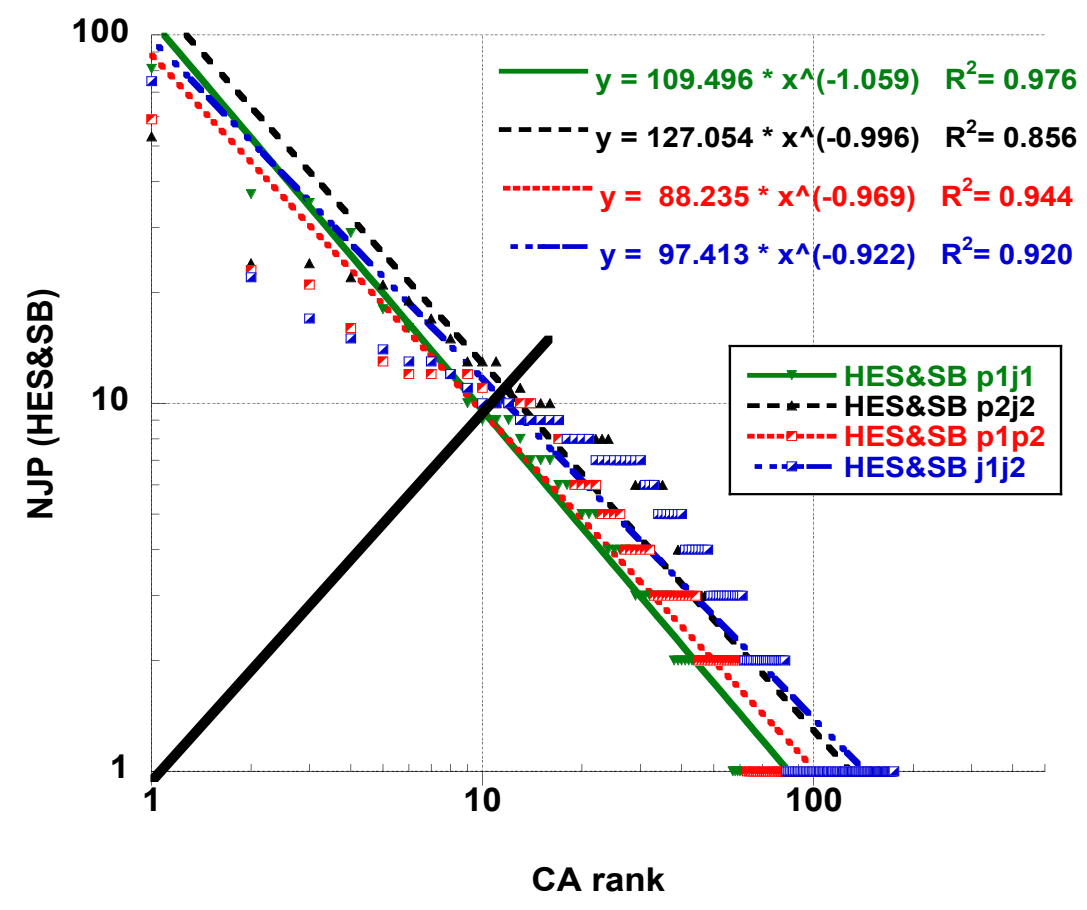


Figure 3: Number of Joint Publications (NJP) by HES\&SH and HES\&SB as a function of the rank of CAs by decreasing importance. Best fits by a power law are shown together with the line indicating the threshold on how to measure the $m_{a} \mathrm{CA}$ core value. This allows to compare the parameters in Eq. 2 for the two main CAs of HES, for the whole set of publications in proceedings (p1p2) or in peer review journals $(\mathrm{j} 1 \mathrm{j} 2)$

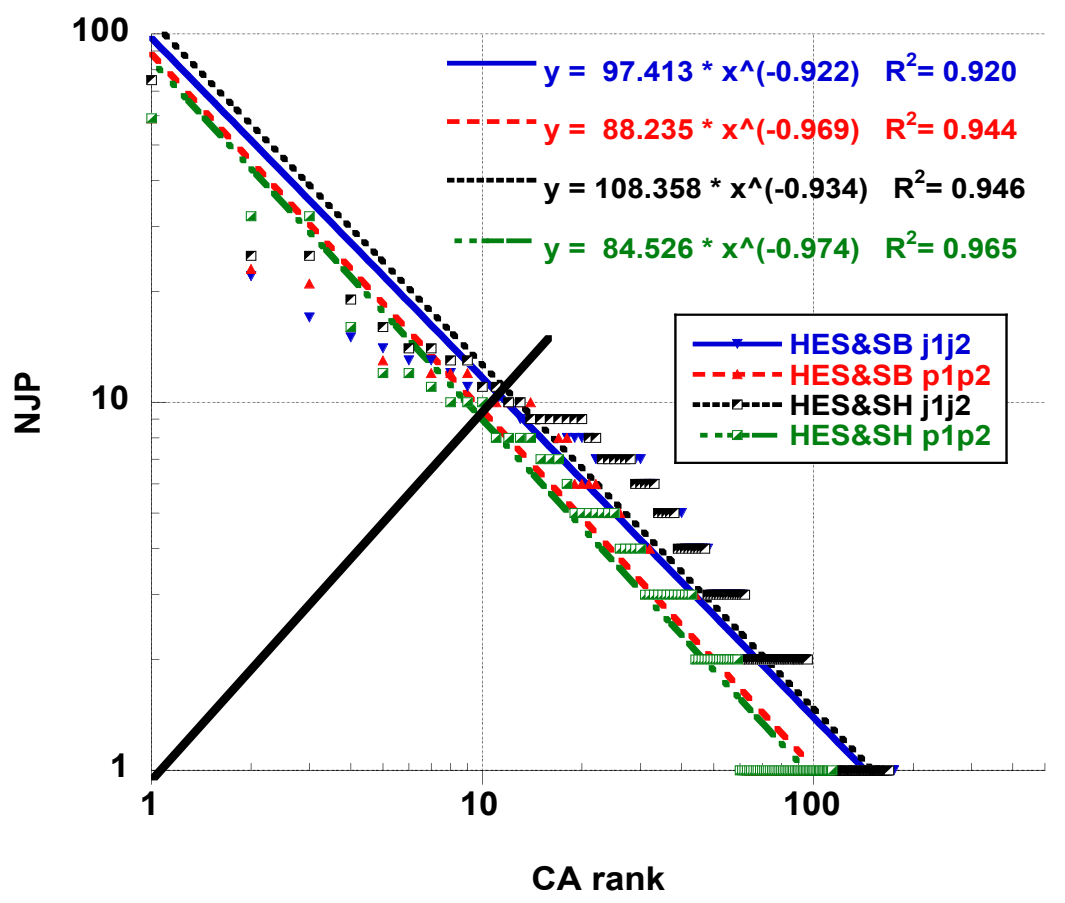


Figure 4: Number of Joint Publications (NJP) by HES\&SH and HES\&SB as a function of the rank of CAs by decreasing importance. Best fits by a power law are shown together with the line indicating the threshold on how to measure the $m_{a} \mathrm{CA}$ core value. This allows to compare the parameters in Eq.22 for the two main CAs of HES, in time intervals 1 and 2 through p1j1 and p2j2

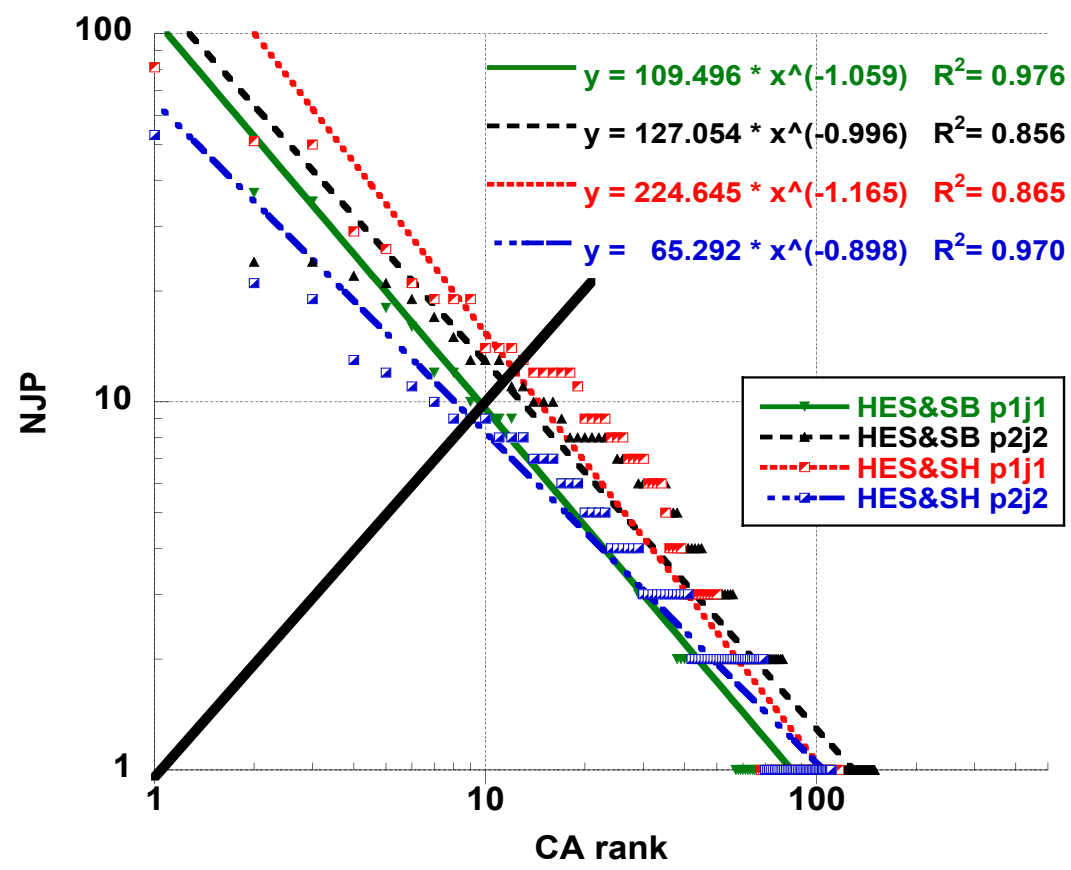


Figure 5: Number of Joint Publications (NJP) by MA\&NV as a function of the rank of CAs by decreasing importance. Best fits by a power law are shown together with the line indicating the threshold on how to measure the $m_{a} \mathrm{CA}$ core value. This allows to compare the value of parameters in Eq. 22 in two time intervals, 1 and 2, through p1j1 and p2j2, as well as to compare the whole set of publications in proceedings (p1p2) with the whole set of papers in peer review journals $(\mathrm{j} 1 \mathrm{j} 2)$

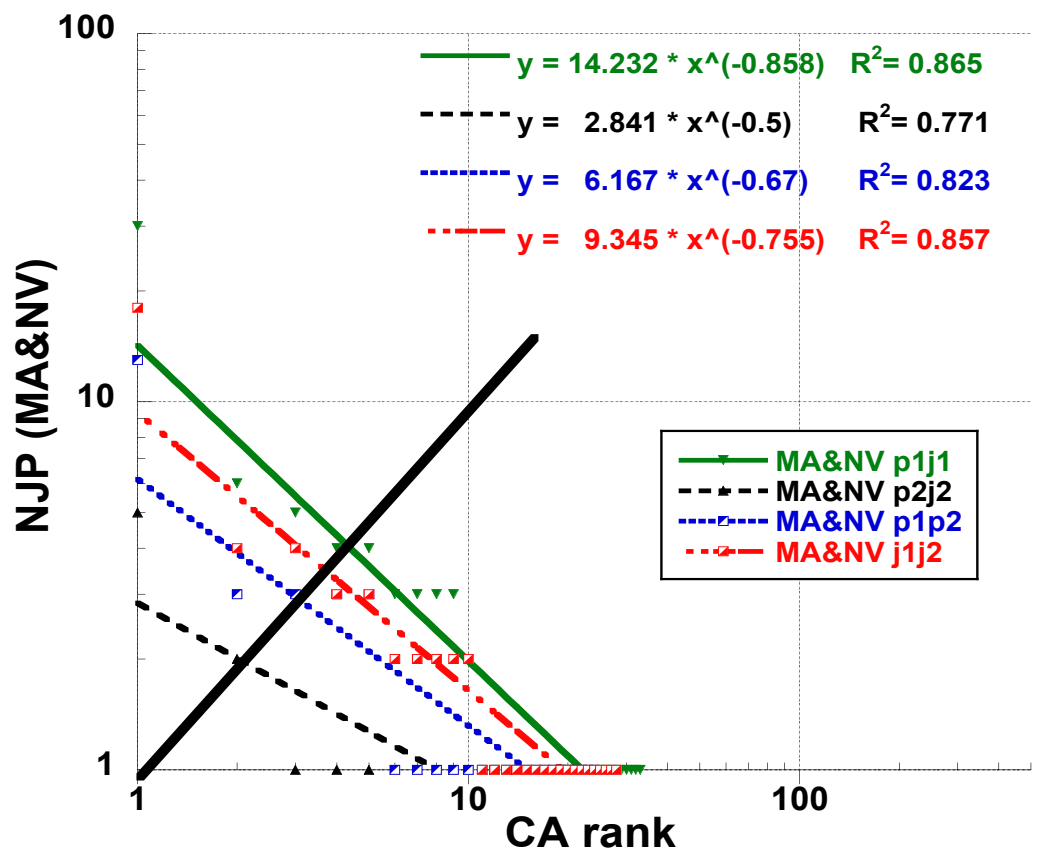


Figure 6: Number of Joint Publications (NJP) by MA\&RC and MA\&NV as a function of the rank of CAs by decreasing importance. Best fits by a power law are shown together with the line indicating the threshold on how to measure the $m_{a}$ CA core value. This allows to compare the parameters in Eq. 2 for the two main CAs of MA, for two time intervals with publications either in proceedings (p1p2) or in peer review journals $(\mathrm{j} 1 \mathrm{j} 2)$

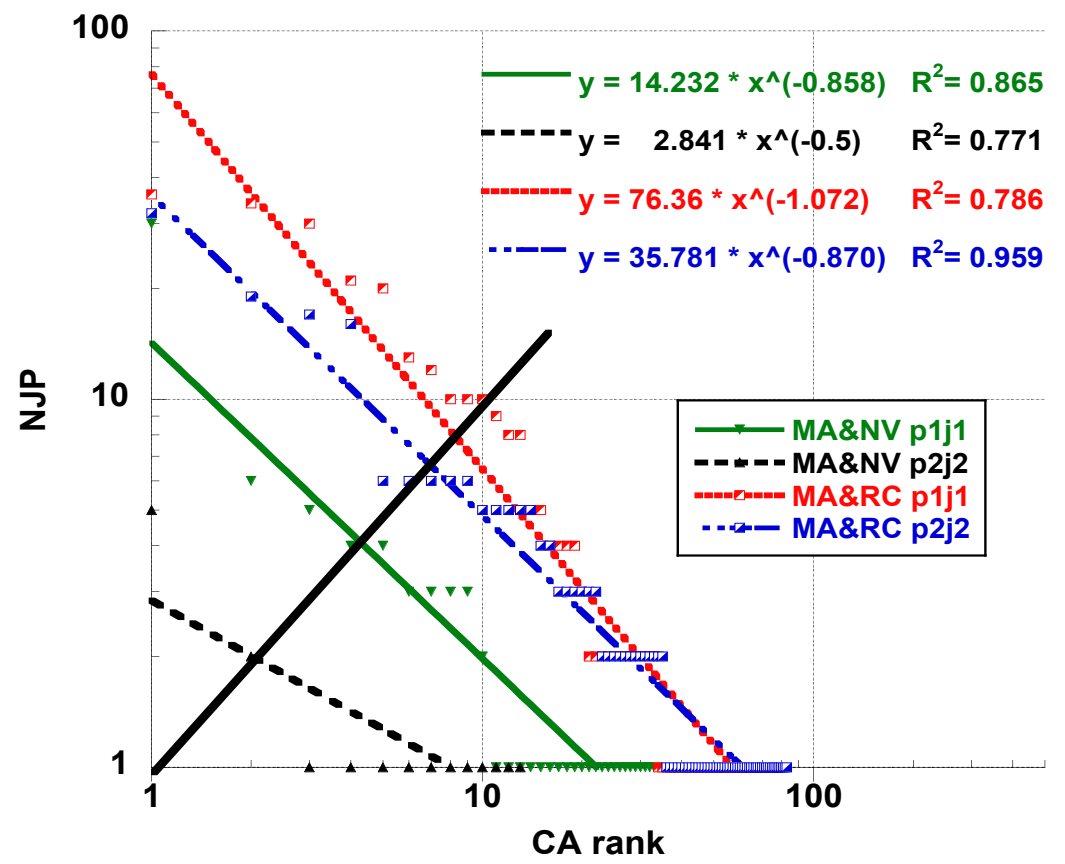


Figure 7: Number of Joint Publications (NJP) by MA\&RC and MA\&NV as a function of the rank of CAs by decreasing importance. Best fits by a power law are shown together with the line indicating the threshold on how to measure the $m_{a}$ CA core value. This allows to compare the parameters in Eq. 20 for the two main CAs of MA, for the whole set of publications either in proceedings (p1p2) or in peer review journals $(\mathrm{j} 1 \mathrm{j} 2)$

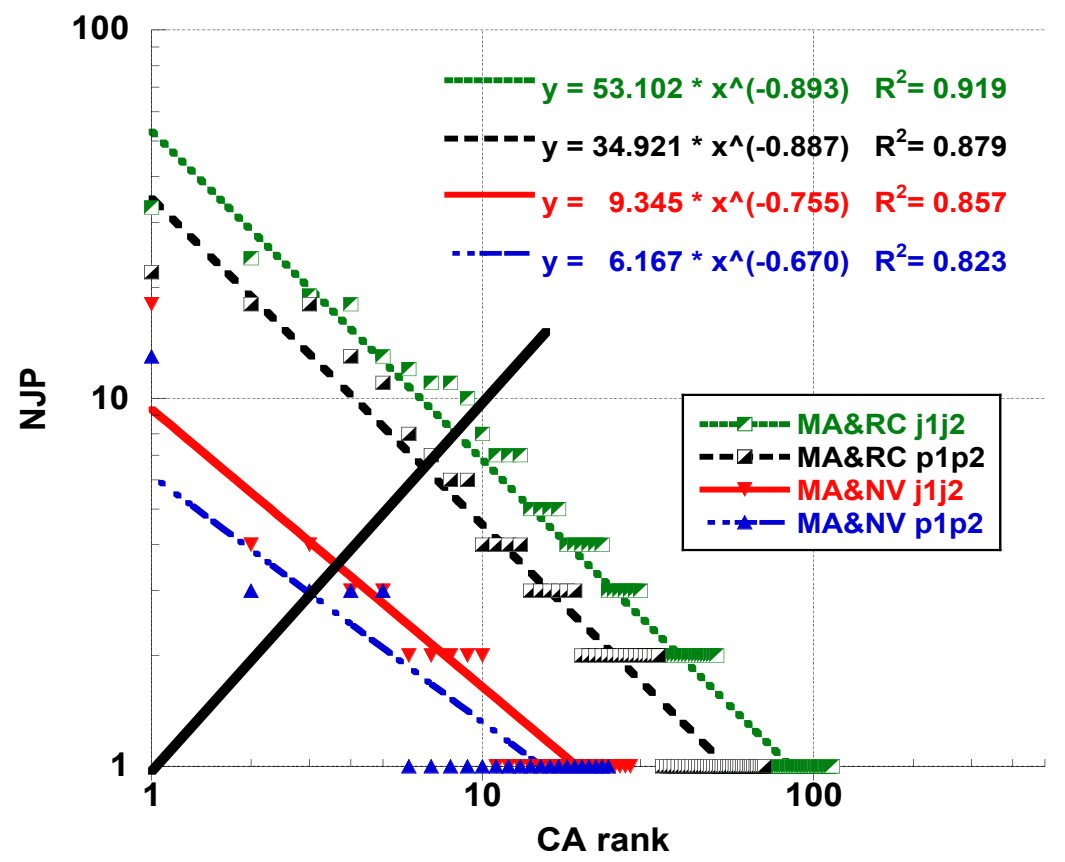


Figure 8: Search for a theoretical relationship, based on a Price-like model Fernández-Cano et al. (2004), between statistical characteristics of BSS CA distributions, i.e. $\mu$ and $\sum$ as a function of $r_{M}$ (三NDCA); the power law and the exponential (Price-like model) fits are shown

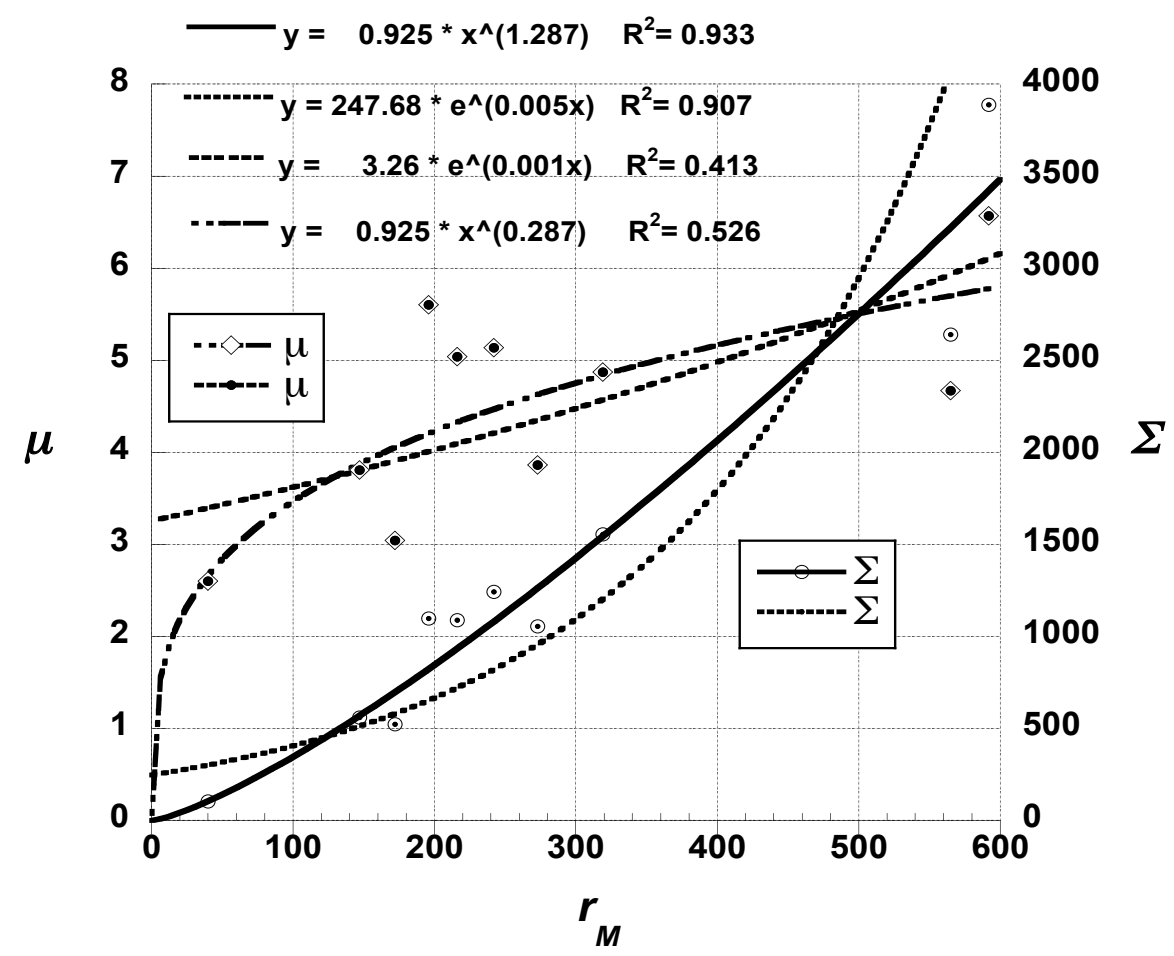

